05

\title{
Флексомагнитный и флексоантиферромагнитный эффекты в центроантисимметричных антиферромагнетиках
}

\author{
(С) А.Ф. Кабыченков, Ф.В. Лисовский \\ Фрязинский фрилиал Института радиотехники и электроники им. В.А. Котельникова РАН, \\ 141190 Фрязино, Московская обл., Россия \\ Ie-mail: lisf@df.ru
}

Поступило в Редакцию 1 октября 2018 г.

В окончательной редакции 1 октября 2018 г.

Принято к публикации 19 ноября 2018 г.

$\mathrm{C}$ позиций магнитной симметрии показано, что центроантисимметричные антиферромагнетики допускают возможность существования в них линейных флексомагнитного и флексоантиферромагнитного эффектов, заключающихся в наведении намагниченности градиентом упругих напряжений или градиентом антиферромагнитного момента соответственно.

DOI: 10.21883/JTF.2019.07.47795.351-18

\section{Введение}

Хотя среди всех типов магнитоупорядоченных сред антиферромагнетики (АФМ) составляют самую многочисленную группу, насчитывающую около двух тысяч химических элементов, соединений и сплавов [1], они до недавнего времени практически не находили сколь-нибудь заметного технического применения. Интересно, что скептически к использованию АФМ относился и Л. Неель (лауреат нобелевской премии 1970 г. „за фундаментальные работы и открытия в области антиферромагнетизма и ферромагнетизма, нашедшие важные применения в физике твердого тела“ [2], отметивший в своей нобелевской лекции, что „они чрезвычайно интересны с теоретической точки зрения, но вряд ли будут где-либо применены“ [3]). С тех пор ситуация мало изменилась, если не принимать во внимание АФМ со слабым ферромагнетизмом, например, ортоферриты, которые занимают достойное место среди магнитооптических устройств для связи и вычислительной техники в качестве управляемых транспарантов, дефлекторов, изоляторов и коммутаторов для волоконно-оптических линий передачи (см., например, [4]).

Такая точка зрения на АФМ была широко распространенной и казалась вполне оправданной, поскольку для создания реальных устройств (например, запоминающих) на основе какой-либо среды, необходимо, чтобы эта среда воспринимала определенные внешние воздействия и реагировала на них таким образом, чтобы отклик можно было зарегистрировать извне. Традиционные методы, используемые в магнитных элементах и устройствах на ферро- и ферримагнетиках, основанные на воздействии на среду внешними магнитными полями различного типа, в рассматриваемом случае неприменимы, поскольку напряженность внешних полей должна быть сопоставима с мощными эффективными магнитными полями обменного происхождения, обеспечивающими антиферромагнитное упорядочение. Кроме того, регистрировать отклик среды по магнитным полям отклика практически невозможно, так как АФМ-полей рассеяния не создает.

Имеется возможность реализации взаимодействия с антиферромагнитной средой через однородные немагнитные воздействия, например, через электрические поля или упругие напряжения при использовании линейных магнитоэлектрического или пьезомагнитного эффектов, существование которых с позиций магнитной симметрии было предсказано в кристаллах, группа симметрии которых не содержит операции обращения времени. Операция обращения времени в таких кристаллах входит в группу симметрии только в комбинации с операциями вращения и (пространственной) инверсии. В частности, магнитоэлектрический эффект может существовать в антиферромагнетиках с центром антисимметрии (антиинверсии), а пьезомагнетизм - в антиферромагнетиках без центра антиинверсии [5-10]. Впервые магнитоэлектрический эффект экспериментально был обнаружен в $\mathrm{Cr}_{2} \mathrm{O}_{3}$ [11-13], а пьезомагнитный в $\mathrm{MnF}_{2}, \mathrm{CoF}_{2}$ и $\mathrm{FeF}_{2}$ [14].

К сожалению, из-за малых значений констант линейной связи между электрической или упругой подсистемами и магнитной подсистемой для получения заметного отклика последней необходима огромная напряженность электрического поля или сильные упругие напряжения. Использование неоднородных воздействий существенно облегчает задачу, поскольку локальное создание больших градиентов электрического поля или упругих напряжений особых затруднений не вызывает.

В настоящей работе в рамках магнитной симметрии рассматриваются неоднородные флексомагнитный и флексоантиферромагнитный эффекты в центроантисимметричных антиферромагнетиках. Такие антиферромагнетики не обладают слабыми ферромагнетизмом и пьезомагнетизмом, которые могут приводить к маскированию изучаемых эффектов. 


\section{Флексомагнитный эфффект}

Флексомагнитный $(\Phi \mathrm{M})$ эффект состоит в наведении намагниченности градиентом упругих напряжений. Он описывается в удельном термодинамическом потенциале слагаемым, линейным по компонентам магнитного поля Н и градиента упругих напряжений $\sigma_{k l}$, т. е.

$$
\Phi^{(f m)}=-\gamma_{i j k l} H_{i} \frac{\partial \sigma_{k l}}{\partial x_{j}},
$$

где $\Phi^{(f m)}-$ слагаемое термодинамического потенциала, отвечающее за возникновение флексомагнитного эффекта, а $\gamma_{i j k l}$ - тензор, симметричный по индексам $k l$.

Из определения магнитной индукции $B_{j}^{(f m)}=-4 \pi \frac{\partial \Phi^{(f m)}}{\partial H_{j}}$ следует, что при $\mathbf{H}=0$ линейная по градиенту напряжений намагниченность составляет

$$
M_{i}^{(f m)}=\gamma_{i j k l} \frac{\partial \sigma_{k l}}{\partial x_{j}}
$$

Обратный эффект состоит в наведении упругих деформаций $u_{k l}^{(f m)}$ градиентом магнитного поля. Используя определение тензора деформаций, находим, что

$$
u_{k l}^{(f m)}=-\frac{\delta \Phi^{(f m)}}{\delta \sigma_{k l}}=-\left(\frac{\partial \Phi^{(f m)}}{\partial \sigma_{k l}}-\frac{\partial}{\partial x_{n}} \frac{\partial \Phi^{(f m)}}{\left(\partial \Phi^{(f m)} / \partial x_{n}\right)}\right),
$$

откуда следует, что

$$
u_{k l}^{(f m)}=\frac{\partial\left(\gamma_{i n k l} H_{i}\right)}{\partial x_{n}} .
$$

Тензор $\gamma_{i n k l}$ нечетен по отношению к операциям обращения времени и пространственной инверсии, поэтому флексомагнитный эффект отсутствует в кристаллах, магнитные группы симметрии которых содержат эти операции отдельно, но может существовать в кристаллах, точечные магнитные группы которых содержат операцию центроантиинверсии (произведение операций обращения времени и пространственной инверсии). Для кубических АФМ эту операцию содержат магнитные группы $m^{\prime} 3, m^{\prime} 3 m$ и $m^{\prime} 3 m^{\prime}$; для тетрагональных группы $4 / m^{\prime}, 4^{\prime} / m /, 4 / m^{\prime} m^{\prime} m^{\prime}$ (например, $\mathrm{Fe}_{2} \mathrm{TeO}_{6}$ ), $4 / m^{\prime} m m$ и $4 / m^{\prime} m^{\prime} m$; для гексагональных - группы $\overline{3}^{\prime}$, $\overline{3}^{\prime} m^{\prime}$ (например, $\left.\mathrm{Cr}_{2} \mathrm{O}_{3}\right), \overline{3}^{\prime} m, 6^{\prime} / m, 6 / m^{\prime}, 6^{\prime} / m m m^{\prime}$, $6 / m^{\prime} m^{\prime} m^{\prime}$ и $6 / m^{\prime} m m$; для ромбических - группы $m^{\prime} m^{\prime} m^{\prime}$ и mmm $^{\prime}$ (например, $\mathrm{Cr}_{2} \mathrm{TeO}_{6}, \mathrm{Cr}_{2} \mathrm{WO}_{6}, \mathrm{~V}_{2} \mathrm{WO}_{6}$ ), для моноклинных - группы $2 / m^{\prime}$ и $2^{\prime} / m[15,16]$.

Из (1) следует, что для кристалла $\mathrm{Cr}_{2} \mathrm{O}_{3}$ отвечающее за возникновение флексомагнитного эффекта слагаемое термодинамического потенциала дается выражением

$$
\begin{aligned}
& \Phi^{(f m)}=-\gamma_{33} H_{z} \frac{\partial \sigma_{z z}}{\partial z}-\gamma_{11}\left[H_{x}\left(\frac{\partial \sigma_{x x}}{\partial x}+\frac{\partial \sigma_{x y}}{\partial y}\right)\right. \\
& \left.+H_{y}\left(\frac{\partial \sigma_{y y}}{\partial y}+\frac{\partial \sigma_{x y}}{\partial x}\right)\right]-\gamma_{12}\left[H_{x}\left(\frac{\partial \sigma_{y y}}{\partial x}-\frac{\partial \sigma_{x y}}{\partial y}\right)\right. \\
& \left.+H_{y}\left(\frac{\partial \sigma_{x x}}{\partial y}+\frac{\partial \sigma_{x y}}{\partial x}\right)\right]-\gamma_{13}\left(H_{x} \frac{\partial \sigma_{z z}}{\partial x}+H_{y} \frac{\partial \sigma_{z z}}{\partial y}\right) \\
& -2 \gamma_{88}\left(H_{x} \frac{\partial \sigma_{x z}}{\partial z}+H_{y} \frac{\partial \sigma_{y z}}{\partial z}\right)-\gamma_{31} H_{z}\left(\frac{\partial \sigma_{x x}}{\partial z}+\frac{\partial \sigma_{y y}}{\partial z}\right) \\
& -2 \gamma_{55} H_{z}\left(\frac{\partial \sigma_{x z}}{\partial x}+\frac{\partial \sigma_{y z}}{\partial y}\right)-2 \gamma_{24}\left[H_{y}\left(\frac{\partial \sigma_{y z}}{\partial y}-\frac{\partial \sigma_{x z}}{\partial x}\right)\right. \\
& \left.-H_{x}\left(\frac{\partial \sigma_{x z}}{\partial y}+\frac{\partial \sigma_{y z}}{\partial x}\right)\right]-\gamma_{42}\left[H_{y}\left(\frac{\partial \sigma_{y y}}{\partial z}-\frac{\partial \sigma_{x x}}{\partial z}\right)\right. \\
& \left.-2 H_{x} \frac{\partial \sigma_{x y}}{\partial z}\right]-\gamma_{72} H_{z}\left[\left(\frac{\partial \sigma_{y y}}{\partial y}-\frac{\partial \sigma_{x x}}{\partial y}\right)-2 \frac{\partial \sigma_{x z}}{\partial x}\right] .
\end{aligned}
$$

Здесь и далее в тензорах 4-го ранга использована сокращенная запись индексов, а именно $11 \rightarrow 1,22 \rightarrow 2$, $33 \rightarrow 3,23 \rightarrow 4,31 \rightarrow 5,12 \rightarrow 6,32 \rightarrow 7,13 \rightarrow 8,21 \rightarrow 9$.

Из (4) следует, что компоненты наводимой градиентом упругих напряжений намагниченности $\mathbf{M}^{(f m)}$ составляют

$$
\begin{aligned}
& M_{x}^{(f m)}=\gamma_{11}\left(\frac{\partial \sigma_{x x}}{\partial x}+\frac{\partial \sigma_{x y}}{\partial y}\right)+\gamma_{12}\left(\frac{\partial \sigma_{y y}}{\partial x}-\frac{\partial \sigma_{x y}}{\partial y}\right) \\
& +\gamma_{13} \frac{\partial \sigma_{z z}}{\partial x}+2 \gamma_{88} \frac{\partial \sigma_{x z}}{\partial z}-2 \gamma_{24}\left(\frac{\partial \sigma_{x z}}{\partial y}+\frac{\partial \sigma_{y z}}{\partial x}\right) \\
& -2 \gamma_{42} \frac{\partial \sigma_{x y}}{\partial z}, \\
& M_{y}^{(f m)}=\gamma_{11}\left(\frac{\partial \sigma_{y y}}{\partial y}+\frac{\partial \sigma_{x y}}{\partial x}\right)+\gamma_{12}\left(\frac{\partial \sigma_{x x}}{\partial y}+\frac{\partial \sigma_{x y}}{\partial y}\right) \\
& +\gamma_{13} \frac{\partial \sigma_{z z}}{\partial y}+2 \gamma_{88} \frac{\partial \sigma_{y z}}{\partial z}+2 \gamma_{24}\left(\frac{\partial \sigma_{y z}}{\partial y}-\frac{\partial \sigma_{x z}}{\partial x}\right) \\
& +\gamma_{42}\left(\frac{\partial \sigma_{y y}}{\partial z}-\frac{\partial \sigma_{x x}}{\partial z}\right), \\
& M_{z}^{(f m)}=\gamma_{33} \frac{\partial \sigma_{z z}}{\partial z}+\gamma_{31}\left(\frac{\partial \sigma_{x x}}{\partial z}+\frac{\partial \sigma_{y y}}{\partial z}\right)+2 \gamma_{55} \\
& \times\left(\frac{\partial \sigma_{x z}}{\partial x}+\frac{\partial \sigma_{y z}}{\partial y}\right)+\gamma_{72}\left[\left(\frac{\partial \sigma_{y y}}{\partial y}-\frac{\partial \sigma_{x x}}{\partial y}\right)-2 \frac{\partial \sigma_{x z}}{\partial x}\right]
\end{aligned}
$$


а компоненты тензора деформаций могут быть представлены в виде

$$
\begin{aligned}
u_{x x}^{(j m)}= & -\frac{\partial\left(\gamma_{11} H_{x}\right)}{\partial x}-\frac{\partial\left(\gamma_{12} H_{y}\right)}{\partial y}-\frac{\partial\left(\gamma_{31} H_{z}\right)}{\partial z}+\frac{\partial\left(\gamma_{42} H_{y}\right)}{\partial z} \\
& +\frac{\partial\left(\gamma_{72} H_{z}\right)}{\partial y}, \\
u_{y y}^{(j m)}= & -\frac{\partial\left(\gamma_{11} H_{y}\right)}{\partial y}-\frac{\partial\left(\gamma_{12} H_{x}\right)}{\partial x}-\frac{\partial\left(\gamma_{31} H_{z}\right)}{\partial z}-\frac{\partial\left(\gamma_{42} H_{z}\right)}{\partial y} \\
& -\frac{\partial\left(\gamma_{72} H_{z}\right)}{\partial y}, \\
u_{z z}^{(j m)}= & -\frac{\partial\left(\gamma_{33} H_{z}\right)}{\partial z}-\frac{\partial\left(\gamma_{13} H_{x}\right)}{\partial x}-\frac{\partial\left(\gamma_{13} H_{y}\right)}{\partial y} \\
u_{x y}^{(j m)}= & u_{y x}^{(j m)}=-\frac{\partial\left(\gamma_{11} H_{x}\right)}{\partial y}-\frac{\partial\left(\gamma_{11} H_{y}\right)}{\partial x}+\frac{\partial\left(\gamma_{12} H_{x}\right)}{\partial y} \\
& +\frac{\partial\left(\gamma_{12} H_{y}\right)}{\partial x}+2 \frac{\partial\left(\gamma_{42} H_{x}\right)}{\partial z}, \\
& +2 \frac{\partial\left(\gamma_{24} H_{x}\right)}{\partial y}+2 \frac{\partial\left(\gamma_{72} H_{z}\right)}{\partial x}, \\
u_{y z}^{(j m)}=u_{z y}^{(j m)}=-2 \frac{\partial\left(\gamma_{88} H_{y}\right)}{\partial z}-2 \frac{\partial\left(\gamma_{55} H_{z}\right)}{\partial y} & u_{z x}^{(j m)}=-2 \frac{\partial\left(\gamma_{88} H_{x}\right)}{\partial z}-2 \frac{\partial\left(\gamma_{55} H_{z}\right)}{\partial x}+2 \frac{\partial\left(\gamma_{24} H_{y}\right)}{\partial x} \\
u_{x z}^{(j m)}+2 \frac{\partial\left(\gamma_{24} H_{x}\right)}{\partial x} . & \\
& -2 y
\end{aligned}
$$

При постоянных коэффициентах деформации будут линейно связаны с градиентом компонент магнитного поля. Так, например, цилиндр из одноосного антиферромагнетика, стоящий вертикально (вертикаль коллинеарна вектору антиферромагнетизма) на жестком основании и находящийся в однородном поле тяготения с гравитационной постоянной $g$ за счет неоднородных деформаций, создаваемых собственным весом, приобретает намагниченность

$$
M_{z}^{(f m)}=\gamma_{33} \frac{\partial \sigma_{z z}}{\partial z}=\gamma_{33} \rho g
$$

где $\rho$ - плотность материала антиферромагнетика.

Аналогичный эффект будет наблюдаться и в случае жесткого закрепления верхнего торца цилиндра на горизонтальной поверхности. При подвешивании цилиндра на упругой нити благодаря приобретенному при намагничивании вращательному механическому импульсу, равному $\mathbf{M}^{(f m)} / \gamma^{(g)}$, где $\gamma^{(g)}-$ гиромагнитный коэффициент, будет происходить поворот антиферромагнитного цилиндра (аналог эффекта Эйнштейна-де Хааза для ферромагнетиков).

Сходные явления могут иметь место и в другой геометрии. При изгибе тонких антиферромагнитных пластин с параллельной нейтральной поверхности базисной плоскостью неоднородные упругие напряжения наводят следующие компоненты намагниченности:

$$
\begin{gathered}
M_{x}^{(f m)}=2 \gamma_{42} \frac{E}{1+\sigma_{P}} \frac{\partial^{2} \varsigma}{\partial x \partial y}, \\
M_{y}^{(f m)}=-\gamma_{42} \frac{E}{1+\sigma_{P}}\left(\frac{\partial^{2} \varsigma}{\partial y^{2}}-\frac{\partial^{2} \varsigma}{\partial x^{2}}\right), \\
M_{z}^{(f m)}=-\gamma_{31} \frac{E}{1-\sigma_{P}}\left(\frac{\partial^{2} \varsigma}{\partial x^{2}}+\frac{\partial^{2} \varsigma}{\partial y^{2}}\right),
\end{gathered}
$$

где $\varsigma(x, y)$ - координаты нейтральной поверхности [17], $E$ - модуль растяжения (модуль Юнга), $\sigma_{P}$ - отношение поперечного сжатия к продольному растяжению (коэффициент Пуассона). Заметим, что появление намагниченности можно легко обнаружить, возбуждая в пластине упругие волны.

\section{Флексоантиферромагнитный эффект}

Флексоантиферромагнитный (ФАМ) эффект состоит в наведении намагниченности градиентом вектора антиферромагнитного момента. Он описывается в термодинамическом потенциале слагаемым, линейным по компонентам магнитного поля Н и градиента антиферромагнитного момента, т.е.

$$
\Phi^{(\text {fam })}=-\eta_{i j k} H_{i} \frac{\partial L_{j}}{\partial x_{k}} .
$$

Потенциал (8) приводит к появлению магнитной индукции, а при отсутствии магнитного поля - намагниченности, равной

$$
M_{i}^{(f a m)}=\eta_{i j k} \frac{\partial L_{j}}{\partial x_{k}},
$$

которая в отличие от слабого ферромагнетизма [5] пропорциональна градиенту антиферромагнитного момента, который может быть обусловлен неоднородностью состава, наличием доменных границ или поверхности раздела кристалла с окружающим пространством, градиентом температуры и т. д. Ответственные за существование ФАМ-эффекта слагаемые термодинамического потенциала для $\mathrm{Cr}_{2} \mathrm{O}_{3}$ имеют вид

$$
\begin{aligned}
& \Phi^{(\text {fam })}=-\eta_{111}\left(H_{x}\left(\frac{\partial L_{x}}{\partial x}-\frac{\partial L_{y}}{\partial y}\right)-H_{y}\left(\frac{\partial L_{x}}{\partial y}+\frac{\partial L_{y}}{\partial x}\right)\right) \\
& -\eta_{123}\left(H_{x} \frac{\partial L_{y}}{\partial z}-H_{y} \frac{\partial L_{x}}{\partial z}\right)-\eta_{231}\left(H_{y} \frac{\partial L_{z}}{\partial x}-H_{x} \frac{\partial L_{z}}{\partial y}\right) \\
& -\eta_{312} H_{z}\left(\frac{\partial L_{x}}{\partial y}-\frac{\partial L_{y}}{\partial x}\right),
\end{aligned}
$$

а намагниченность составляет

$$
\begin{aligned}
M_{x}^{(f a m)}= & \eta_{111}\left(\frac{\partial L_{x}}{\partial x}-\frac{\partial L_{y}}{\partial y}\right)+\eta_{123} \frac{\partial L_{y}}{\partial z}-\eta_{231} \frac{\partial L_{z}}{\partial y} \\
M_{y}^{(f a m)}= & \eta_{111}\left(\frac{\partial L_{x}}{\partial y}-\frac{\partial L_{y}}{\partial x}\right)-\eta_{123} \frac{\partial L_{x}}{\partial z}-\eta_{231} \frac{\partial L_{z}}{\partial x} \\
& M_{z}^{(\text {fam })}=-\eta_{312}\left(\frac{\partial L_{x}}{\partial y}-\frac{\partial L_{y}}{\partial x}\right) .
\end{aligned}
$$


Неоднородность температуры в плоскости $(x, y)$ приводит к появлению компонент намагниченности $M_{x}^{(f a m)}=-\eta_{231} \frac{\partial L_{z}}{\partial y}$ и $M_{y}^{(\text {fam })}=-\eta_{231} \frac{\partial L_{z}}{\partial x}$.

$\mathrm{B}$ присутствии внешнего магнитного поля $\mathbf{H}_{0}$ полный термодинамический потенциал следует записывать в виде

$$
\Phi=\Phi_{0}-\mathbf{M H}_{0}+\left(\mathbf{H}_{m}^{2} / 8 \pi\right),
$$

где $\mathbf{H}_{m}-$ поле, создаваемое намагниченностью [1]. В квадратичном приближении по $\mathbf{L}$ и $\mathbf{M}$ и производным L (производные $\mathbf{M}$ из-за малости не учитываются) термодинамический потенциал $\mathrm{Cr}_{2} \mathrm{O}_{3}$ с учетом ответственных за ФАМ-эффект слагаемых равен

$$
\begin{aligned}
& \Phi_{0}=\frac{1}{2}\left[a_{1}^{(l)} \mathbf{L}^{2}+a_{1}^{(m)} \mathbf{M}^{2}+a_{1}^{(l n)}\left(\frac{\partial \mathbf{L}}{\partial x_{i}}\right)^{2}+a_{2}^{(l)} L_{z}^{2}\right. \\
& \left.+a_{2}^{(m)} M_{z}^{2}+a_{2}^{(l n)}\left(\frac{\partial L_{z}}{\partial x_{i}}\right)^{2}\right]-\eta_{1}\left[M_{x}\left(\frac{\partial L_{x}}{\partial x}-\frac{\partial L_{y}}{\partial y}\right)\right. \\
& \left.-M_{y}\left(\frac{\partial L_{x}}{\partial y}+\frac{\partial L_{y}}{\partial x}\right)\right]-\eta_{2}\left(M_{x} \frac{\partial L_{y}}{\partial z}-M_{y} \frac{\partial L_{x}}{\partial z}\right) \\
& -\eta_{3}\left(M_{y} \frac{\partial L_{z}}{\partial x}-M_{x} \frac{\partial L_{z}}{\partial y}\right)-\eta_{4} M_{z}\left(\frac{\partial L_{x}}{\partial y}-\frac{\partial L_{y}}{\partial x}\right),
\end{aligned}
$$

а процедура минимизации потенциала с использованием условий $\delta \Phi / \delta \mathbf{M}=0$ и $\delta \Phi / \delta \mathbf{L}=0$ приводит к следующей системе уравнений:

$$
\begin{gathered}
M_{x}=\eta_{1}^{(m)}\left(\frac{\partial L_{z}}{\partial y}-\frac{\partial L_{y}}{\partial y}\right)+\eta_{2}^{(m)} \frac{\partial L_{y}}{\partial z} \\
-\eta_{3}^{(m)} \frac{\partial L_{y}}{\partial z}+\xi_{\perp} H_{x}, \\
M_{y}=-\eta_{1}^{(m)}\left(\frac{\partial L_{x}}{\partial y}+\frac{\partial L_{y}}{\partial x}\right)-\eta_{2}^{(m)} \frac{\partial L_{x}}{\partial z} \\
+\eta_{3}^{(m)} \frac{\partial L_{z}}{\partial x} y+\xi_{\perp} H_{y}, \\
M_{z}=\eta_{4}^{(m)}\left(\frac{\partial L_{x}}{\partial y}-\frac{\partial L_{y}}{\partial x}\right)+\zeta_{\|} H_{z}, \\
L_{x}+a_{11}^{(l n)} \frac{\partial^{2} L_{x}}{\partial x_{i}^{2}}-\eta_{1}^{(l)}\left(\frac{\partial M_{x}}{\partial x}-\frac{\partial M_{y}}{\partial y}\right) \\
+\eta_{2}^{(l)} \frac{\partial M_{y}}{\partial z}-\eta_{4}^{(l)} \frac{\partial M_{z}}{\partial y}=0, \\
L_{z}+a_{12}^{(l n)} \frac{\partial^{2} L_{y}}{\partial x_{i}^{2}}+\eta_{3}^{(l)}\left(\frac{\partial M_{x}}{\partial y}-\frac{\partial M_{y}}{\partial x}\right)=0 \\
L_{y}+a_{11}^{(l n)} \frac{\partial^{2} L_{y}}{\partial x_{i}^{2}}+\eta_{1}^{(l)}\left(\frac{\partial M_{x}}{\partial y}+\frac{\partial M_{y}}{\partial x}\right) \\
M_{x} \\
\eta_{4}^{(l)} \frac{\partial M_{z}}{\partial x}=0,
\end{gathered}
$$

где $\eta_{i}^{(m)}=\eta_{i} / a_{1}^{(m)}$ для $i=1,2,3 ; \eta_{4}^{(m)}=\eta_{4} /\left(a_{1}^{(m)}+a_{2}^{(m)}\right)$; $\eta_{j}^{(m)}=-\eta_{j} / a_{1}^{(l)}$ для $j=1,2,4 ; \eta_{3}^{(l)}=-\eta_{3} /\left(a_{1}^{(l)}+a_{2}^{(l)}\right)$; $a_{11}^{(l n)}=-a_{1}^{(l n)} / a_{1}^{(l)} ; a_{12}^{(l n)}=-\left(a_{1}^{(l n)}+a_{2}^{(l n)}\right) /\left(a_{1}^{(l)}+a_{2}^{(l)}\right)-$ приведенные константы, $\xi_{\perp}=1 / a_{1}^{(m)} ; \xi_{\|}=1 /\left(a_{1}^{(m)}+a_{2}^{(m)}\right)$; $\mathbf{H}=\mathbf{H}_{0}+\mathbf{H}_{m}-$ внутреннее магнитное поле.

Однородное состояние типа „легкая ось“ в отсутствие внешнего магнитного поля реализуется при отрицательных константах однородного обмена $a_{1}^{(l)}$ и анизотропии $a_{2}^{(l)}$ и положительной константе неоднородного обмена $a_{1}^{(l n)}$. В этом случае при малой неоднородности антиферромагнитного момента $\left(\left(\eta_{i}^{(m)} / d\right) L \ll \xi H\right.$ и $a_{11}^{(l n)} / d^{2} \ll 1$, где $d$ - масштаб неоднородности) градиент магнитного поля наводит следующие компоненты антиферромагнитного момента:

$$
\begin{gathered}
L_{x}=\chi_{1}^{(l)}\left(\frac{\partial H_{x}}{\partial x}-\frac{\partial H_{y}}{\partial y}\right)+\chi_{2}^{(1)} \frac{\partial H_{z}}{\partial y} ; \\
L_{y}=-2 \chi_{1}^{(l)} \frac{\partial H_{x}}{\partial y}-\chi_{2}^{(l)} \frac{\partial H_{z}}{\partial x} ; \quad L_{z}=0,
\end{gathered}
$$

где $\chi_{1}^{(l)}=-\eta_{1}^{(m)} / a_{1}^{|l|}$ и $\chi_{2}^{(l)}=\left(\eta_{2}^{(m)}-\eta_{4}^{(m)}\right) / a_{1}^{(l)}$ - обобщенные восприимчивости.

\section{Заключение}

Выполненный в работе теоретический анализ дополняет современные представления о свойствах центроантисимметричных АФМ, которые обычно упоминаются исключительно только как пример сред, обладающих магнитоэлектрическим эффектом, а также о возможностях их практического использования. Последнее облегчаются тем, что флексомагнитный и флексоантиферромагнитный эффекты возникают при наличии неоднородных упругих напряжений или магнитных полей, локальное создание больших градиентов которых особых затруднений не вызывает.

\section{Список литературы}

[1] Ожсогин В.И., Шапиро В.Г. Антиферромагнетики Физические величины / ред. И.С. Григорьев, Е.З. Мелихов. М.: Энергоатомиздат, 1991. 648 с.

[2] Электронный ресурс. Режим доступа: http://www.nobelprize.org/nobel_prizes/physics/laureates/ 1970/neel-facts.html

[3] Электронный ресурс. Режим доступа: http://www.nobelprize.org/nobel_prizes/physics/laureates/ 1970/neel-lecture.pdf

[4] Балбашов А.М., Червоненкис А.Я. Магнитные материалы для микроэлектроники. М.: Энергия, 1979. 216 с.

[5] Ландау Л.Д., Лифиии, М.Е. Электродинамика сплошных сред. М.: ГИТТЛ, 1957. 532 с.

[6] Белов Н.В., Неронова И.Н., Смирнова Т.С. // Труды Института кристаллографии. 1955. Т. 11. С. 33-67.

[7] Тавгер Б.А., Зайцев В.М. // ЖЭТФ. 1956. Т. 30. Вып. 3. C. 564-568. 
[8] Дзялошинский И.Е. // ЖЭТФ. 1957. Т. 33. Вып. 3. C. $881-882$.

[9] Birss R.R. Symmetry and Magnetism. Amsterdam: North-Holland, 1964. 252 p.

[10] Сиротин Ю.И., Шаскольская М.П. Основы кристаллофизики. М.: Наука, 1979. 640 с.

[11] Астров Д.Н. // ЖЭТФ. 1960. Т. 38. Вып. 3. С. 984-985.

[12] Rado G.T., Folen V.J. // Rhys. Rev. Lett. 1961. Vol. 6. N 2. P. 607-608.

[13] Rivera J.-P. // Ferroelectrics. 1994. Vol. 161. N 1. P. 165-180.

[14] Боровик А.С. // ЖЭТФ. 1960. Т. 38. Вып. 4. С. 1088-1098.

[15] Туров Е.A. // УФН. 1994. Т. 164. Вып. 3. С. 325-332.

[16] Туров Е.А., Колчанов А.В., Меньшенин В.В., Мирсаев И.Ф., Николаев В.В. Симметрия и физические свойства антиферромагнетиков. М.: Наука, 2001. 560 с.

[17] Ландау Л.Д., Лифиии Е.M. Теория упругости. М.: Наука, 1987. $248 \mathrm{c}$. 